\title{
Treatment Outcome of Cervical Tear Drop Fracture
}

\author{
Hyeon Jun Kim, Kyu Yeol Lee, Woo Chul Kim \\ Department of Orthopedic Surgery, Dong-A University College of Medicine, Busan, Korea
}

\begin{abstract}
Study Design: This is a retrospective study.
Purpose: We wanted to evaluate the clinical results of surgical and conservative treatment for cervical tear drop fracture.

Overview of Literature: The tear drop fracture of the lower cervical spine is generally associated with a high incidence of neurological deficits and surgery is needed to treat this injury. Tear drop fracture of C2 is usually a stable fracture that is amendable to conservative treatment.

Methods: We reviewed the outcomes of 25 patients. Cervical tear drop fracture was classified as the extension and flexion types according to the mechanism of injury. The neurologic symptoms were evaluated by the Frankel classification system, and the loss of lordosis and disc height, and the duration of bony union were analyzed.

Results: Twenty one patients had the flexion type injury and 4 patients had the extension type injury. All the patients with the flexion type were treated by anterior decompression and plate stabilization. All the patients with the extension type were treated conservatively. Ten patients with the flexion type had neurologic deficits. The nerve root injuries recovered fully and the incomplete injuries had an average 1.5 grade recovery. Radiologically, the extension type fracture showed bony union at an average of 12.8 weeks. For the patients with the flexion type fracture, the loss of lordosis was $2.6^{\circ}$ and the loss of disc height was $2.1 \mathrm{~mm}$. The period of bony union in 20 cases was 13.0 weeks.

Conclusions: Anterior plate stabilization was an effective treatment for the flexion type tear drop fracture. Conservative treatment is thought to be one of the good clinical methods for treating the extension type tear drop fracture.
\end{abstract}

Key Words: Cervical spine, Tear drop fracture, Anterior plate stabilization

\section{Introduction}

Cervical spine injury is a severe injury that may lead to tetraplegia or death. Therefore, the importance of treatment and rehabilitation for cervical spine injury has been underscored due to the severe social and economic losses. Satisfactory decompression has not been achieved for cases of cervical tear drop fracture that are treated with skeletal traction by using skull tongs or halo-vest fixation. Despite adequate traction, there are some cases in which the fragment of the vertebral body was displaced to the anterior aspect of the spinal canal on the posterior side of the vertebral body.

Cervical tear drop fracture is divided into two types based on the mechanism of injury, and most of which is flexion type injury. The cases of cervical tear drop fracture of the C2 vertebral body usually manifest as a hyperextension type injury. The anterior longitudinal ligament originates from the base of $\mathrm{C} 2$ (the axis). Accordingly, in cases of extension injury, the avulsion fracture occurs at the bottom of the anteroinferior side of the vertebral body. This can be differentiated from a tear drop fracture of the other lower cervical

Received Aug 18, 2009; 1st revised Sep 9, 2009; accepted Sep 10, 2009

Corresponding author: Lee Kyu Yeol, MD

Department of Orthopedic Surgery, Dong-A University College of Medicine,

Dongdaesin-dong 3-ga, Seo-gu, Busan 602-715, Korea

Tel: +82-51-240-2867, Fax: +82-51-243-9764, E-mail: gylee@dau.ac.kr 
spines. In this tear drop fracture of the vertebral body of $\mathrm{C} 2$ (the axis), the stability of the construction of the posterior side is maintained. Surgery is rarely needed for cases of tear drop fracture of $\mathrm{C} 2$ vertebral body. The treatment is decided upon by differentiating these cases from cases of the flexion type tear drop fracture (Fig. 1).

For effective decompression of the spinal cord in cases of the flexion type injury, corpectomy for the fractured vertebral body has been proposed by several authors ${ }^{1,2}$.

Instead of removing the vertebral body, anterior corpectomy and fusion, where the ilium or fibula is transplanted may induce the occurrence of graft dislodgment or the disappearance of the realignment in cases in which injury of the posterior longitudinal ligament is present. Delayed kyphotic angulation may also occur. The methods for resolving these problems include prolonged skeletal traction following the surgery or fixation via combined anterior \& posterior stabilization. Another alternative method has been suggested to be anterior decompression with plate fixation ${ }^{3-5}$

In the current study, we retrospectively analysed the radiological changes and clinical results in cases of cervical tear drop fracture that were treated conservatively or with surgery.

\section{Materials and Methods}

We retrospectively analysed 25 patients for whom a follow-up study could be performed for more than one year following the onset of cervical tear drop fracture, and these patients were treated at out hospital form May 1998 to May 2007. There were 21 cases of the flexion type and four cases of the extension type. All the cases of the flexion type underwent anterior plate fixation using cervical locking plates (Medtronics ${ }^{\circledR}$ plate) via an anterior surgical approach (Fig. 2).

The mean patient age was 43.2 years (range, 17 to 71 years) and the male-to-female ratio was 20:5. The mean follow-up period was 18 months (range, 12 to 41 months). The mechanisms of injury include ten cases of road traffic accident, eight cases of falling down, four cases of slipping down and three cases of other causes. These results indicate that the cervical injury due to traffic accidents was the most prevalent. The sites of injury included four cases of $\mathrm{C} 2$, seven cases of $\mathrm{C} 4$, nine cases of $\mathrm{C} 5$, four cases of $\mathrm{C} 6$ and one case of $\mathrm{C} 7$.

In accordance with the mechanism of injury, four cases of $\mathrm{C} 2$ had an extension type tear drop fracture and the remaining 21 cases had a flexion type tear drop fracture.


Fig. 1. A 27 year old female patient with a C2 tear drop fracture. (A) The initial lateral roentgenogram shows a displaced antero-inferior bony fragment. (B) The lateral radiograph obtained 3 months after trauma shows consolidation of the fractured site. 
The flexion type tear drop fractures were classified into four types according to the system of Korres et al. ${ }^{6}$, which is based on the size of the anterior inferior fragment and the displacement of the posterior part of the vertebral body into the spinal canal. In Type I, there was rupture of the posterior ligament and the characteristics of a small fracture $(<3$ $\mathrm{mm}$ in size) on the anterior inferior angle of the vertebral body, half of the vertebral body lacked sagittal fracture at the posterior body and retrolisthesis was seen. In Type II, there were characteristics of the coronary fracture of the anterior inferior angle of the vertebral body, a lack of the retrolisthesis and there was sagittal fracture of the posterior
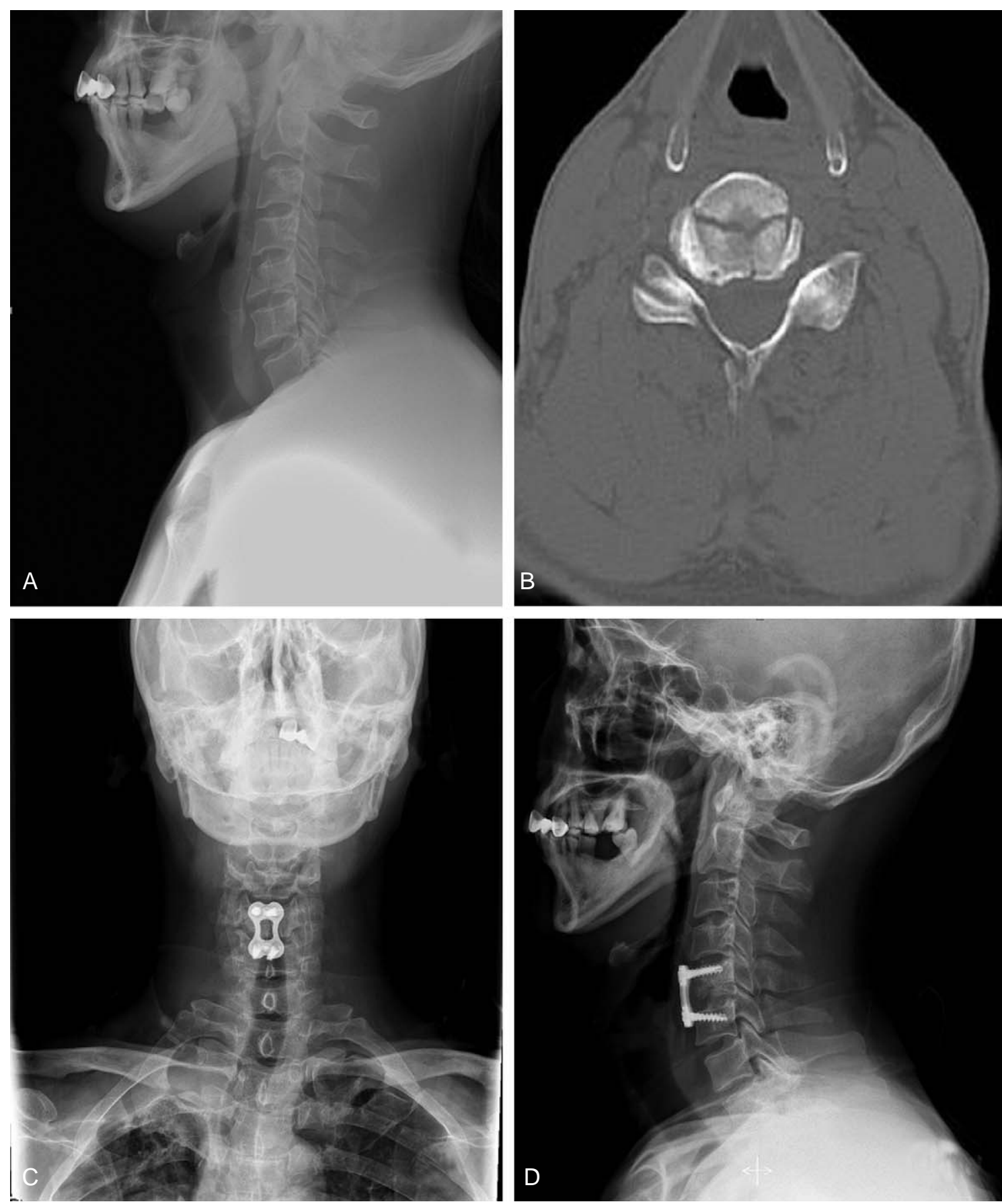

Fig. 2. A 52 year old male patient with a C5 tear drop fracture (Case No.14). (A) The preoperative lateral roentgenogram shows anterior displacement of a bony fragment. (B) The CT shows a T-shape pattern with a sagittal fracture. (C) The postoperative radiograph shows the consolidation of the grafted bone. (D) The postoperative radiograph shows the consolidation of the grafted bone. 
body. In Type III, a subclassification is done into two subtypes, a and b, based on the vertebral body or the retrolisthesis of its fragments. Those cases in which the displacement was greater than $4 \mathrm{~mm}$ corresponded to type IIIb and those cases in which the displacement was smaller than 4 $\mathrm{mm}$ corresponded to type IIIa. Type IV represents the anterior inferior angle of the vertebral body associated with posterior facet dislocation and anterior dislocation of the vertebrae above. Allen et al. ${ }^{7}$ classified flexion injury into the compression and flexion injuries and the extension and flexion injuries. For the cases of compression and flexion injury, Stage I represents impaction of the vertebral body where there was a lack of injury of the posterior ligament, Stage II represents a loss of the height of the anterior column and beaking of the anterior body, Stage III represents an oblique direction of the fracture line to the anterior side of the vertebral body through the inferior subchondral plate, Stage IV represents the displacement of fracture and an inferoposterior bone fragment into the spinal canal of $<3$ $\mathrm{mm}$ and Stage IV represents the presence of injury of the posterior longitudinal ligament and retrolisthesis. Based on the classification system of Korres et al. ${ }^{6}$, Type I corresponds to Stage III of Allen. Type II and Type IIIa are close to Stage IV of Allen. Cases of > Type IIIb correspond to StageV. Yet due to the difference in the radiologic criteria there is a discrepancy to some extent between the 2 classification systems. In the current study, for the classification system for a tear drop fracture, we used Korres's classification and based on this, radiologic comparison can be easily made.

A clinical evaluation of the neurologic severity was based on Frankel's functional classification. Radiogically, a simple X-ray was taken postoperatively and at last follow-up to evaluate for a loss of lordosis, a loss of disc height, the duration of bony union. The case of graft displacement and metal failure were also reviewed.

\section{Results}

For the cases of the flexion type, there were nine cases of C5 and this was the most prevalent. There were seven cases of $\mathrm{C} 4$, four cases of $\mathrm{C} 6$ and one case of $\mathrm{C} 7$. In the cases of $\mathrm{C} 2$ corresponding to the extension type, the fracture was confirmed in four cases. Conservative treatment was performed in four cases of the extension type tear drop fracture. Radiogically, during a mean period of 12.8 weeks (range, 11.3 to 16.2 weeks), there were findings that were suggestive of bony union. But there were no findings suggestive of a neurologic deficit and postoperative complications.

For the cases of flexion type tear drop fracture, there were six cases of Type I, seven cases of Type II, three cases of Type IIIa, three cases of Type IIIb, and two cases of Type IV. Of these, there were ten cases of neurologic deficits. There were five cases of complete spinal cord injury (one case of Type II, one case of Type IIIa, two cases of Type IIIb and one case of Type IV), four cases of incomplete spinal cord injury (one case of Type II, one case of Type IIIa, one case of Type IIIb, and one case of Type IV) and one case of nerve root injury (one case of Type I) (Table 1). Generally, conservative treatment was performed in the Type I cases in which there was no concurrent presence of neurologic deficits. In our series, conservative treatment was performed at the initial phase of the outpatient visits. Thereafter, for monitoring the clinical course, a follow-up observation was performed via a dynamic flexion extension lateral X-ray. Operation was then performed if the findings indicated instability.

There were no cases in which the postoperative neurologic deficits were aggravated. The nerve root injuries fully recovered in all the cases. The incomplete spinal cord injuries showed an average grade of recovery of 1.5 , in

Table 1. Type of injury and neurological status

\begin{tabular}{|c|c|c|c|c|c|c|c|}
\hline \multirow{2}{*}{ Type } & \multirow{2}{*}{ No. } & \multicolumn{5}{|c|}{ Frankel's classification } & \multirow{2}{*}{ Neurologic deficit $(\%$} \\
\hline & & A & $\mathrm{B}$ & $\mathrm{C}$ & $\mathrm{D}$ & $E$ & \\
\hline Extension & 4 & 0 & 0 & 0 & 0 & 4 & 0 \\
\hline Flexion & 21 & 5 & 1 & 3 & 1 & 11 & 47.6 \\
\hline I & 6 & 0 & 0 & 0 & 1 & 5 & 16.7 \\
\hline II & 7 & 1 & 0 & 1 & 0 & 5 & 28.6 \\
\hline IIIa & 3 & 1 & 0 & 1 & 0 & 1 & 66.7 \\
\hline IIIb & 3 & 2 & 1 & 0 & 0 & 0 & 100 \\
\hline IV & 2 & 1 & 0 & 1 & 0 & 0 & 100 \\
\hline Total & 25 & 5 & 1 & 3 & 1 & 15 & 40.0 \\
\hline
\end{tabular}


accordance with Frankel's classification.

On the simple lateral X-rays, the mean loss of lordosis was $2.0^{\circ}\left(1.5^{\circ}\right.$ to $\left.2.4^{\circ}\right)$ in the cases of Type I, it was $2.3^{\circ}$ $\left(1.6^{\circ}\right.$ to $\left.3.1^{\circ}\right)$ in the cases of Type II, $2.8^{\circ}\left(2.5^{\circ}\right.$ to $\left.3.0^{\circ}\right)$ in the cases of Type IIIa, $3.1^{\circ}\left(2.5^{\circ}\right.$ to $\left.3.5^{\circ}\right)$ in the cases of Type IIIb and $4.45^{\circ}\left(4.2^{\circ}\right.$ to $\left.4.7^{\circ}\right)$ in the cases of Type IV. The loss of disc height was $1.2 \mathrm{~mm}(0.5$ to $1.8 \mathrm{~mm})$ in the cases of Type I, $2.2 \mathrm{~mm}$ (1.1 to $3.5 \mathrm{~mm}$ ) in the cases of Type II, $2.1 \mathrm{~mm}$ (1.8 to $2.3 \mathrm{~mm}$ ) in the cases of Type IIIa, $2.5 \mathrm{~mm}$ (2.1 to $3.2 \mathrm{~mm}$ ) in the cases of Type IIIb and 4.2 $\mathrm{mm}$ (4.1 to $4.2 \mathrm{~mm})$ in the cases of Type IV. Overall, the mean loss of lordosis was $2.6^{\circ}\left(1.5^{\circ}\right.$ to $\left.4.7^{\circ}\right)$ and the mean loss of disc height was $2.1 \mathrm{~mm}(0.5 \mathrm{~mm}$ to $4.2 \mathrm{~mm})$. With excluding one case of Type IV, all the remaining cases had bone union with a high union rate of $92 \%$. The duration for bony union was a mean of 13.0 weeks (11.4 to 16.4 weeks) (Table 2).

In regard to the complications, there were no cases of displacement of the graft or metal failure. There were no cases of complications such as postoperative infection or iatrogenic nerve injury. There was nonunion in one case of Type
IV. There was one case of the intermittent dysphagia. Following the accident, in one case in which there was a complete spinal cord injury, death occurred due to respiratory complications at three months postoperatively.

\section{Discussion}

With the increased occurrence of traffic accidents and industrial disasters, cervical spinal cord injury due to cervical spine fracture and dislocation may leave severe complications such as quadriplegia where there is almost no chance for rehabilitation. For these patients, it is important that treatment and rehabilitation be stated early. According to Durbin ${ }^{8}$ and Jacob $^{9}$, spinal cord injury frequently occurs in young active male patients, and the major causes have been reported to be traffic and industrial accidents. Also in our series, 20 of the 25 patients were men. The mean age was 43.2 years and this is a relatively young age. The causes of injury included ten cases of traffic accident and this accounted for $40 \%$ of the total cases. The common sites of

Table 2. Data on 25 patients with tear drop fracture of the cervical spine

\begin{tabular}{|c|c|c|c|c|c|c|}
\hline $\begin{array}{r}\text { Case } \\
\text { No. }\end{array}$ & Age/Sex & $\begin{array}{l}\text { Type of } \\
\text { fracture }\end{array}$ & $\begin{array}{c}\text { Loss of } \\
\text { Lordosis }\left(^{\circ}\right)\end{array}$ & $\begin{array}{c}\text { Loss of } \\
\text { disc height }(\mathrm{mm})\end{array}$ & $\begin{array}{c}\text { Duration of } \\
\text { bony union (wk) }\end{array}$ & $\begin{array}{c}\text { Neurologic change } \\
\text { (Frankel's classification) }\end{array}$ \\
\hline 1 & $29 / F$ & IIIb & 2.5 & 2.1 & 15.8 & 0 \\
\hline 2 & $63 / \mathrm{M}$ & II & 1.7 & 2.5 & 12.5 & 0 \\
\hline 3 & $50 / \mathrm{M}$ & I & 2 & 1 & 11.4 & 0 \\
\hline 4 & $48 / \mathrm{M}$ & Extension & & & 11.8 & 0 \\
\hline 5 & $47 / \mathrm{M}$ & II & 2.3 & 1.8 & 12.3 & 0 \\
\hline 6 & $69 / \mathrm{M}$ & I & 2 & 1.8 & 12 & 1 \\
\hline 7 & $22 / \mathrm{M}$ & II & 2.7 & 1.9 & 11.4 & 0 \\
\hline 8 & $27 / F$ & Extension & & & 11.8 & 0 \\
\hline 9 & $50 / \mathrm{M}$ & IIIa & 2.9 & 1.8 & 15.2 & 1 \\
\hline 10 & $47 / \mathrm{M}$ & IV & 4.7 & 4.2 & Nonunion & 0 \\
\hline 11 & $30 / \mathrm{M}$ & IV & 4.2 & 4.1 & 16.4 & 2 \\
\hline 12 & 49/M & Extension & & & 16.2 & 0 \\
\hline 13 & $25 / \mathrm{M}$ & I & 1.5 & 0.5 & 12.1 & 0 \\
\hline 14 & $52 / \mathrm{M}$ & II & 3.1 & 1.1 & 12.4 & 0 \\
\hline 15 & $47 / \mathrm{M}$ & IIIb & 3.3 & 3.2 & 13.3 & 0 \\
\hline 16 & $71 / \mathrm{F}$ & I & 2.4 & 0.8 & 12.7 & 0 \\
\hline 17 & $47 / \mathrm{F}$ & $\mathrm{IIIb}$ & 3.5 & 2.1 & 13 & 2 \\
\hline 18 & $43 / \mathrm{M}$ & I & 1.9 & 1.3 & 12.9 & 0 \\
\hline 19 & 39/M & Extension & & & 11.3 & 0 \\
\hline 20 & $28 / \mathrm{M}$ & II & 2.1 & 2.2 & 12.1 & 1 \\
\hline 21 & $25 / \mathrm{M}$ & IIIa & 2.5 & 2.3 & 14.4 & 0 \\
\hline 22 & $59 / \mathrm{F}$ & II & 2.5 & 3.5 & 11.7 & 0 \\
\hline 23 & $17 / \mathrm{M}$ & IIIa & 3 & 2.3 & 13.4 & 0 \\
\hline 24 & $38 / \mathrm{M}$ & II & 1.6 & 2.6 & 11.8 & 0 \\
\hline 25 & $59 / \mathrm{M}$ & I & 2.1 & 1.7 & 12.3 & 0 \\
\hline
\end{tabular}


injury included C5 and this was the most prevalent in our study. The reasons for this are that the cervical spine anatomically formsed a lordotic angle and the stress is concentrated on C5 during cervical flexion ${ }^{10,11}$. Also in our series, damage to $\mathrm{C} 5$ was seen in nine cases and this accounted for $36 \%$ of the total cases. Due to the cervical instability because of cervical tear drop fracture, manual reduction or open reduction and operative treatment were performed to prevent spinal cord injury, further neural damage was prevented and adequate stabilization was obtained. Thus, early mobilization of the patients became possible and this helped prevent complications that are due to a longterm period of bed rest, such as pulmonary infection, urinary infection, compressive sores, gastrointestinal problems and/or psychiatric problems, in addition to making the nursing \& rehabilitation easier. These points could be the goals of treatment for cases of cervical spinal cord injury. There are several treatment methods for cases of injury to the lower cervical spine. The complications that follow operative treatment can be avoided for the cases that receive conservative treatment. Yet due to the necessity for a long period of bed rest \& immobilization, such complications as pulmonary and urinary infection, compressive sore and contracture of the extremities may occur. When instability and non-union are seen, there is a disadvantage that operative treatment may be needed ${ }^{12,13}$. By contrast, there is a tendency that operative treatment has frequently been performed in recent years based on the advantages such as stabilizing the cervical spine, early ambulation, a shortened period of admission, the relative ease of the nursing $\&$ treatment and the improvement of mental health ${ }^{14,15}$.

An anterior cervical approach that was proposed by Smith and Robinson ${ }^{16}$ and Bailey and Badgley ${ }^{17}$, has increasingly been done for the past 25 years. An appropriate decompression of the spinal canal has become a useful surgical modality for patients with cervical tear drop fracture. Based on the results of our current study, this surgical procedure could not alter the neurologic recovery of complete cord injured patients. This might have a significant effect on the recovery of incomplete cord injured patients. For the cases of the flexion type of cervical tear drop fracture, there is often the concurrent presence of rupture of the posterior longitudinal ligament. Early dislodgment of the graft may be induced with a single use of an anterior surgical approach. This may eventually lead to instability. Further, kyphotic angulation may also occur following surgery ${ }^{18}$. To resolve these problems, such treatment modalities as prolongation of skeletal traction, combined anterior and posterior stabilization and the anterior decompression with plate fixation have been performed.

Skeletal traction could not produce satisfactory treatment outcomes because of the psychiatric and physiologic effects that were due to the prolonged bed rest period. A concomitant use of the anterior and posterior surgical approaches was not desirable because of the increased rate of morbidity for the additional operation.

According to Cabanela and Ebersold ${ }^{19}$, following the spinal decompression via an anterior surgical approach, anterior plate fixation was the most useful, safe treatment method for the cases of unstable flexible cervical tear drop fracture. It has long been debated whether there is a discrepancy of the quality of the results between the anterior surgical approach and the posterior surgical approach for cases of cervical fracture ${ }^{20}$. The fixation and fusion via an anterior surgical approach can be performed with the patient in a supine position. For posterior fixation, for cases of spinal cord injury due to position change or for multi-level cord injured patients, there was difficulty in selecting the surgical positioning of the patient. There was less risk of damaging the soft tissue with using an anatomical approach, and maintaining the normal cervical curvature was also possible. The fusion rate can be raised according to the location of the compressive site of the graft. With the removal of the intervertebral disc or body, there is an advantage that extensive anterior decompression becomes possible ${ }^{21}$. As compared with the posterior fixation or combined anterior and posterior fixation, anterior fixation alone in cervical spine fracture is hard to get a significant stability and difficult to reduce the dislocation that was not reduced manual reduction $^{22}$.

For the cases of cervical flexible tear drop fracture, plate fixation via an anterior surgical approach might be a more useful modality. For cases of cervical tear drop fracture, there is compression to the anterior part of the vertebral body in the anterior region of the spinal canal. This is not only because the posterior segment of the body can be removed without the risk of developing spinal cord injury, but also because the extruded disc can also be easily removed.

For the cases of the extension type cervical tear drop fracture, according to the classification system of Allen, when the retrolisthesis due to the injury of the posterior ligament was present, then surgical treatment was also recommended. In our series, there was no instability noted on the sim- 
ple lateral X-rays and the dynamic flexion-extension Xrays. Accordingly, in all the cases, for conservative treatment, the patients were recommended to wear a Philadelphia neck brace for 12 weeks. Medical treatment was performed for the management of clinical symptoms such as neck pain.

\section{Conclusions}

For the cases of the extension type cervical tear drop fracture, the findings suggestive of bone union confirmed that complications would not occur. For the cases of the extension type cervical tear drop fracture, in most of the cases, with excluding the Type IV cases, the anterior plate stabilization using an anterior metal plate might be a useful modality. Yet posterior fixation might also be considered for the cases in which the stability cannot be maintained following anterior interbody fusion or for those Type IV cases.

\section{REFERENCES}

1. Bohlman HH, Boada E: Fractures and dislocations of the lower cervical spine. (in The Cervical Spine Research Society eds. The cervical spine, 2nd ed. Philadelphia, Lippincott: 355,1989 )

2. Pierce DS: Acute treatment of spinal cord injuries. (in Pierce DS, Nickel VH eds. The total care of spinal cord injuries. Boston, Little Brown and Company: 1, 1977)

3. Bohler J, Gaudernak T: Anterior plate stabilization for fracture-dislocations of the lower cervical spine. J Trauma 1980; 20: 203-205.

4. Bremer AM, Nguyen TQ: Internal metal plate fixation combined with anterior interbody fusion in cases of cervical spine injury. Neurosurgery 1983; 12: 649-653.

5. Castaing J: Les traumatismes recents du Raquis cervical inferieur: traitement chirurgical par voie anterieure. Rev Chir Orthop 1984; 70: 519-522.

6. Korres DS, Stamos K, Andreakos A, Spyridonos S, Kavadias K: The anterior inferior angle fracture of a lower cervical vertebra. Eur Spine J 1994; 3: 202-205.

7. Allen BL Jr, Ferguson RL, Lehmann TR, O'Brien RP: A mechanistic classification of closed, indirect fractures and dislocations of the lower cervical spine. Spine (Phila Pa 1976) 1982; 7: 1-27.
8. Durbin FC: Fracture-dislocations of the cervical spine. J Bone Joint Surg Br 1957; 39: 23-38.

9. Jacobs B: Cervical fractures and dislocations (C3-7). Clin Orthop Relat Res 1975; (109): 18-32.

10. Norrell H, Wilson CB: Early anterior fusion for injuries of the cervical portion of the spine. JAMA 1970; 214: 525530.

11. Penning L: Normal movements of the cervical spine. AJR Am J Roentgenol 1978; 130: 317-326.

12. Burke DC, Berryman D: The place of closed manipulation in the management of flexion-rotation dislocations of the cervical spine. J Bone Joint Surg Br 1971; 53: 165-182.

13. Castellano V, Bocconi FL: Injuries of the cervical spine with spinal cord involvement (myelic fractures): statistical considerations. Bull Hosp Joint Dis 1970; 31: 188-194.

14. Chung JY, Shin HC, Kim HS: Anterior plate fixation of the racture-dislocation of cervical spine. J Korean Orthop Assoc 1988; 23: 1541-1548.

15. Evans DK: Reduction of cervical dislocations. J Bone Joint Surg Br 1961; 43: 552-555.

16. Smith GW, Robinson RA: The treatment of certain cervical-spine disorders by anterior removal of the intervertebral disc and interbody fusion. J Bone Joint Surg Am 1958; 40: 607-624.

17. Bailey RW, Badgley CE: Stabilization of the cervical spine by anterior fusion. J Bone Joint Surg Am 1960; 42: 565-594.

18. Capen DA, Garland DE, Waters RL: Surgical stabilization of the cervical spine: a comparative analysis of anterior and posterior spine fusions. Clin Orthop Relat Res 1985; (196): 229-237.

19. Cabanela ME, Ebersold MJ: Anterior plate stabilization for bursting teardrop fractures of the cervical spine. Spine (Phila Pa 1976) 1988; 13: 888-891.

20. Stauffer ES: Fractures and dislocation of the spine. Part I: The cervical spine. (in Rockwood CA Jr, Green DP eds. Fractures in adults, 2nd ed. Philadelphia, Lippincott: 987, 1984).

21. Aebi M, Zuber K, Marchesi D: Treatment of cervical spine injuries with anterior plating. Indications, techniques, and results. Spine (Phila Pa 1976) 1991; 16: S38- S45.

22. Adams MS, Crawford NR, Chamberlain RH, et al: Biomechanical comparison of anterior cervical plating and combined anterior/lateral mass plating. Spine J 2001; 1 : 166-170. 